\title{
The Upper Beam Tryout Process Combined with Finite Element Simulation and Experiment
}

\author{
YU Qiang ${ }^{1,2,3, a}$ and LIANG Jin ${ }^{1,3,4, b}$ \\ ${ }^{1}$ School of Mechanical Engineering, Xi' an Jiaotong University, ShaanXi 710049, P.R.China; \\ ${ }^{2}$ TianJin Engineering Center of Digital Manufacturing Die and Mold, Tianjin University of \\ Technology and Education, Tianjin 300222, P.R.China; \\ ${ }^{3}$ State Key Laboratory for Manufacturing Systems Engineering, Xi' an Jiao tong University, Shan'xi \\ 710049, P.R.China; ${ }^{1}$ \\ ${ }^{4}$ Guangdong Shunde Xi'an Jiaotong University Academy, guangdong 528000, P.R.China; \\ ayuqiang95@yeah.net, ${ }^{b}$ liangjin@mail.xjtu.edu.cn
}

\begin{abstract}
An upper beam is investigated by combination of finite element simulation and tryout methods. The results of deformation simulation and grid experiment were studied. The results show that the direction of principal and side strain in the crack area simulated by CAE software are same as the results received by grid experiment. The result gives a direction for solving the crack problems. It is important that the blank size in both ends is reduced to decrease the bending stress in the die radius. But the effect is very limited for the end region is far from crack area. Combined with the method of reducing the blank size, decreasing the height of the drawbead, enlarging the die radius, improving the smoothness the tension stress is decreased effectively. And the qualified drawing panel is produced finally.
\end{abstract}

\section{Introduction}

Automobiles have become the important transportation for human. Crash safety has become one of the key points considering for consumers. For improving the safety performance of automobile collision, more and more high strength steels are used in body in white. For high strength steels the yield point is higher than low-carbon steel and the yield ratio is bigger and easier to spring back. Even plastic deformation is happened the tensile strength is reached rapidly to be crack. As a result the panel formability is not easy to ensure[1]. The success rate of formability is effectively improved with finite element software [2-4]. But there are some mistakes sometimes in the finite simulation leading to big differences between real tryout and simulation results. Thus for manufacturing qualified parts the analysis in tryout and corrective methods should be done to solve the problems rapidly.

\footnotetext{
* Corresponding author:yuqiang95@yeah.net
} 


\section{The Analysis of Stamping Process and Finite Element Simulation}

The panel studied in this paper is upper beam in auto body as shown in Fig 1. The panel size is $1040 \times 115 \times 80 \mathrm{~mm}$; the thickness is $1.5 \mathrm{~mm}$ with material $\mathrm{B} 250 \mathrm{P} 1$. The material properties are shown in table 1 . The material has high yield point and is not easy to get plastic deformation. And the springback and wrinkle are easy to happen. The work piece shape is like a bone with a square stepped surface in both sides. There is a slender and narrow surface with wave type. The crack possibility should be considered in the region of surface changed severely. At the same time the plastic deformation should be improved in the flat surface to decrease springback. Based on above consideration the draw surface is designed as shown in Fig 2. For improving the formability of panel the convex in the both ends are designed in order to increase the flow resistance of material from side to inner. Then the tensile stress and plastic deformation nearby area will be increased and the springback will be decreased accordingly. The convex in all the area of the middle place are designed with the same purpose.

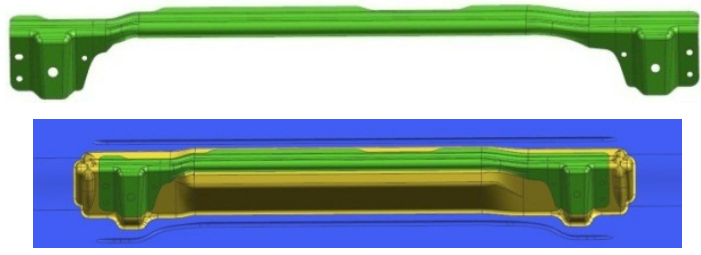

Fig 1 the 3D surface of the panel

Fig 2 the drawing surface of the panel

Table 1 the Material Properties of B250p1

\begin{tabular}{|c|c|c|c|c|c|c|c|c|c|}
\hline Material & $\mathrm{E} / \mathrm{GPa}$ & $v$ & $\sigma s / \mathrm{MPa}$ & $K / \mathrm{MPa}$ & $\sigma b / \mathrm{MPa}$ & $n$ & $r 0$ & $r 45$ & $r 90$ \\
\hline $\mathrm{B} 250 \mathrm{P} 1$ & 207 & 0.28 & 300 & 709 & 451 & 0.19 & 2.31 & 1.41 & 1.84 \\
\hline
\end{tabular}

The drawing process of the panel is simulated with finite element simulation software of blank forming. The material properties used in simulation are shown in table 1 . The friction coefficient is 0.15 and the press process is single action with binder force $9 \times 10^{5} \mathrm{~N}$. The binder stroke is $100 \mathrm{~mm}$. And the virtual drawbead is used in the simulation process. The forming limited diagram (FLD) is show in Fig 3. The FLD shows a good acceptable formability status with no crack point. There are only some wrinkle areas in the drawn panel that can be accepted. With this simulation results the die tryout is carried out after die design and machining.

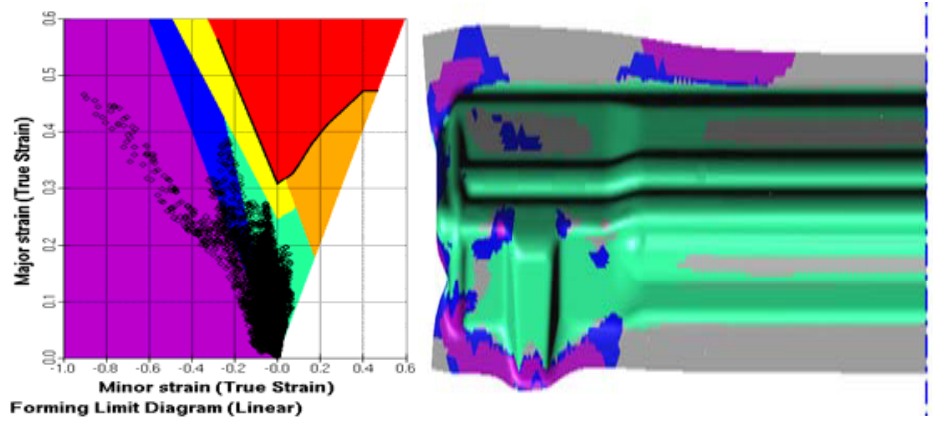

Fig 3 the drawing simulation result 1 


\subsection{The Tryout and Improvement Process of Panel}

According to the CAE simulation results the drawing die tryout is carried out. The blank size is $1170 \times 280 \mathrm{~mm}$. The binder stroke and binder force is the same as simulation. The blank position is fixed with the position simulated in CAE software. The tryout results are shown in Fig 4. The flange portion part is very flat as shown in Fig 4 without a wide range of wrinkling problem predicted in CAE analysis. But there are some cracks in the vertical direction in the step surface. The maximum width of the crack is nearly $8 \mathrm{~mm}$. The tryout result is failed.

According to tryout experience there is little difference between the CAE simulation and actual tryout results generally. But there are large differences between them. The cause of the problem should be found rapidly to solve the crack defect.

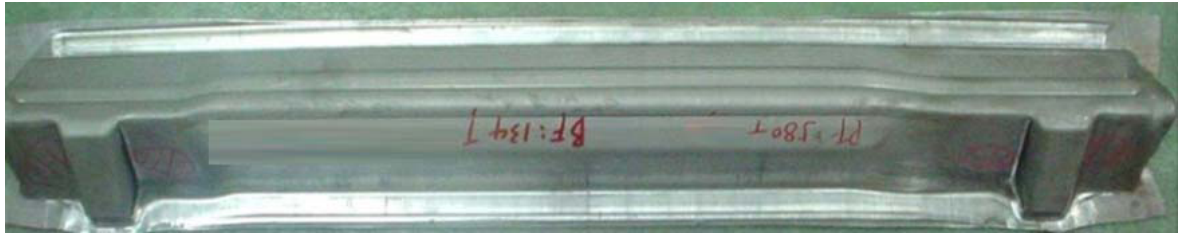

Fig 4 the crack drawing panel after the first tryout process

\subsection{The Problem Analysis and Improvement Process}

The root cause of crack is large tensile stress exceeding the tensile strength. The basic method of solving crack problem is increasing the amount of material to decrease the value of tensile stress. Base on the improvement theory combining with the distribution of primary and secondary stress showed in Fig 5 simulated with FEM software. It is obvious that the material in the direction of 30 degree with the horizontal direction suffers great tensile stress. And the material in the direction of 30 degree with the vertical direction suffers great compressive stress. Comparing the stress value the tensile stress is greater than compressive stress. For the defect here it is crack and the direction of the crack is vertical. The tensile stress is along the direction shown in the Fig 5.

For verifying the accuracy of the finite element calculations the grid Experiment of drawing blank is carried out. The circle grid of $\Phi 5 \mathrm{~mm}$ is printed with the grid printing apparatus, grid paper and electrolyte. The blank drawing process is carried out with the position and blank size simulated by CAE software. The deformation of grids in crack area is shown in Fig 6. The circle grids are stretched obviously in crack area shown in Fig 6. The maximum amount of deformation can reach $184 \%$. The stretching direction is same as the results shown in Fig 4. The experiment results show that the strain direction is correct simulated with finite element software.
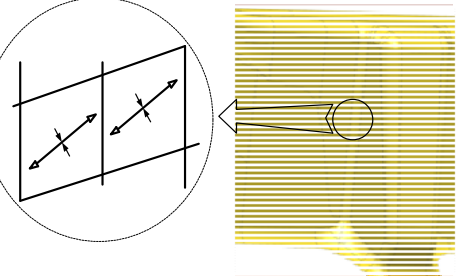

Fig 5 the strain direction of simulation result

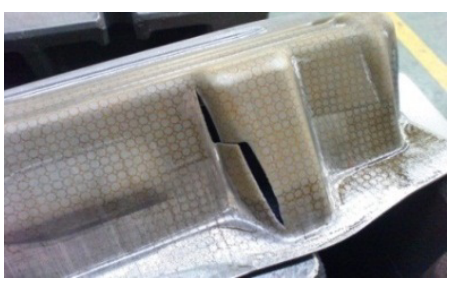

Fig 6 the grid deformation of experiment result

Based on the distribution of strain decreasing the resistance of material flowing is considered to increase the material inflows in crack area. Based on the analysis the blank 
size is decreased $15 \mathrm{~mm}$ in the both ends to decrease the bending resistance when the material flows to the crack area from both ends. The material retention case with the original blank and the blank decreasing $15 \mathrm{~mm}$ in both ends are shown in Fig 7 . When the blank size in one end decreases $15 \mathrm{~mm}$ less material exists shown in Fig 7-b in end than the case with original blank shown in Fig 7-a. The blank bending deformation in ends is less. Thus the material flowing resistance is decreased to have a good impact on the crack area. But the distance from the end to crack area is a little far with $140 \mathrm{~mm}$, so the influence level of decreasing the blank size in ends is difficult to determine. The degree of crack and the width of gap are smaller than before. But the panel is also crack. Thus another method should be carried out to solve the problem.

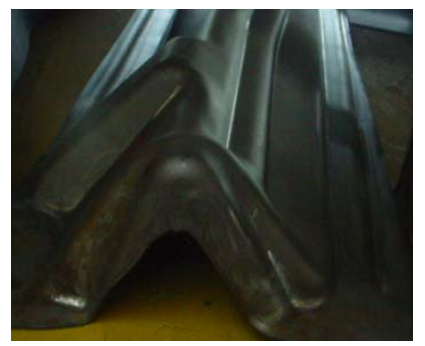

A)

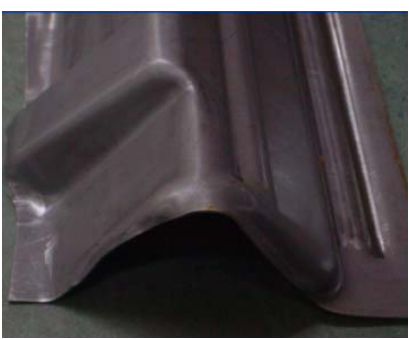

B)

a) The drawing result of original blank size

b) The drawing result of blank decreased $15 \mathrm{~mm}$ one end

Fig 7 the drawing result of blank with different blank size

For reducing the drawing resistance of material on step face shown in fig 5, further another two methods are used to do die tryout shown in Fig 8. In order to reduce the tension stress of material outside the punch opening a notch is added in the left lower corner of step face shown in Fig8-a. Thus the material can flow into the step surface easily. Based on the same reason the drawing radiuses are enlarged in the mark area of the left lower corner of step face Fig8-b. Thus the bending stress of the material flowing into the punch can be reduced. It can be seen from the two pictures that the crack areas are reduced especially the result shown in Fig8-a. And it is obviously that decreasing the blank size outside the punch opening line is more effective.

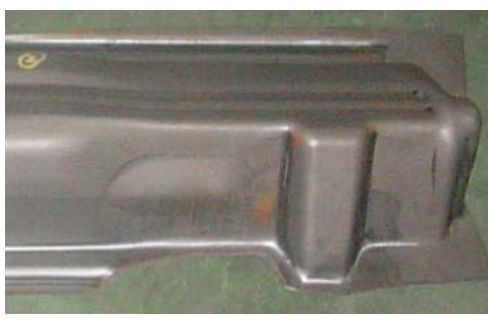

A)

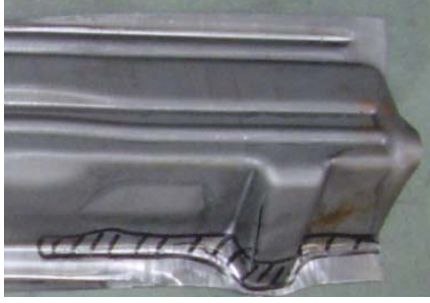

B)

a) Add notch in the left lower corner of step face

b) Enlarge the drawing radius in the left lower corner of step face

Fig 8 two methods of reducing flowing resistance in step face

After the above tryout methods and under the premise of enlarging the radius in the left lower corner of the stepped surface it is effective that reducing the blank size can decrease the crack. But if the blank with notch was used an additional blanking die should be designed to increase the cost of production. In order to achieve the same effect and under 
the premise of enough trim allowance the blank size of the bottom is reduced $10 \mathrm{~mm}$ and the height of drawbead is decreased at the same area which is shown in Fig9. These drawbeads are originally designed to decrease the wrinkle. Reducing friction coefficient is another important method to improve the material flowing. It is good for the material flowing into the internal parts that the binder and die face are polished with whetstone to increase smoothness and decrease the friction coefficient specifically for the die tryout process.

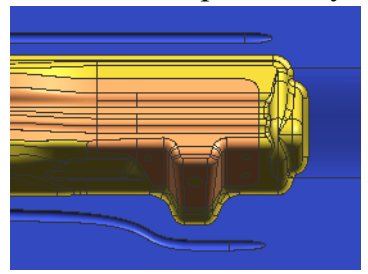

Fig 9 the distribution of drawbead on the end

Under the premise of CAE simulation and grid experiment the methods of reducing the blank size, decreasing the height of the drawbeads, enlarging the die radius and improving the smooth of die face are used to promote the material inflow. The qualified drawing part is obtained with the tension stress decreased effectively which is shown in Fig 10.

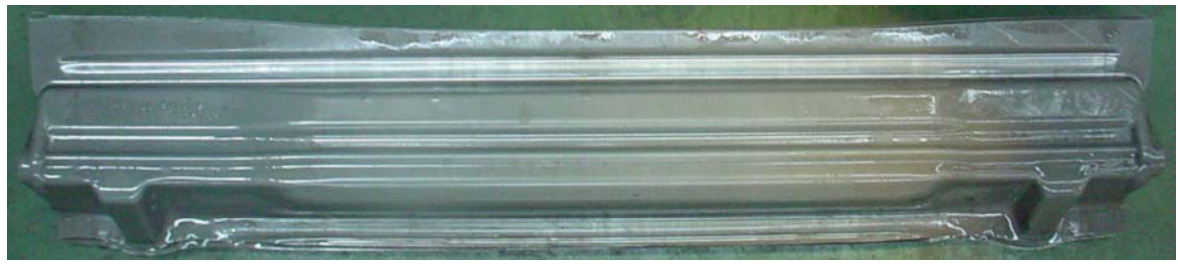

Fig 10 the qualified drawing part

\section{Conclusions}

CAE technology of material forming is very important for increasing the qualified rate of autobody forming, shorten production cycle and decreasing the production cost. In this paper a upper beam is studied with CAE simulation and grid experiment methods. And the die tryout method for improving the panel crack is carried out. Through this research, the followings are summarized and point out.

1. The direction of principal and side strain in the crack area simulated by CAE software is same as the results received by grid experiment. The result gives a direction for solving the crack problems.

2. It is important that the blank size in both ends is reduced to decrease the bending stress in the die radius. But the effect is very limited for the end region is far from crack area.

3. Combined with the method of reducing the blank size, decreasing the height of the drawbead, enlarging the die radius, improving the smoothness the tension stress is decreased effectively. And the qualified drawing panel is produced finally.

\section{Acknowledgments}

This work is supported by Development Program for Outstanding Young Teachers in Tianjin, Tianjin science and technology innovation system and the condition of platform projects, Development Foundation of Tianjin University of Technology and Education Tianjin, China under Grant RC14-39, 14TXGCCX00011,KJ12-13, KJY12-12 respectively. 
This work is also supported by National Natural Science Foundation of China (Grant No. 51421004, 51275378, 51275389) , supported by the Fundamental Research Funds for the Central Universities, supported by Guangdong Public Research and Capacity Building Special Funds (0104 3D body rapid scanning system research)

\section{References}

1. Yu Qiang. The accuracy study of springback prediction for anti-collision beam based on die deformation. Forging \& Stamping Technology, 2013, 38(3):54-57.

2. Zhu Y C, et al. Simulation research of V-bending springback for ultra-high-strength steel sheet. Forging \& Stamping Technology, 2012,37 (5):31-34.

3. Bekar, et al. Robust springback optimization of a dual phase steel seven-flange die assembly. Structural \& Multidisciplinary Optimization, 2012, 46 (3) : 425-444.

4. Abdullah A B, et al. A comprehensive review of experimental approaches used in the measurement of springback. Advances in Natural and Applied Sciences, 2012, 6(2): 195-205. 